\title{
CONFLICT BETWEEN DIRECTIONAL AND CENTRIPETAL SELECTION
}

\author{
J. W. JAMES \\ Department of Animal Husbondry, University of Queensland, Brisbane
}

\section{INTRODUCTION}

Received 3.ii.62

As is well-known, selective pressures may vary at different stages of the life cycle in a population. Perhaps the clearest demonstration of this is that of Lack (1954), who showed that in the English swift, Apus apus, different pressures at egg laying and rearing resulted in selection favouring an intermediate egg number. Much of natural selection does favour intermediate phenotypes, e.g. Rendel (I 943), Karn and Penrose (195I). Mather (I96I) has pointed out that though there is presumably some optimum phenotype for adaptation to the physical environment, competition between members of a population may lead to directional selection, though, as he showed, it leads to centripetal selection for sternopleural bristles in Drosophila melanogaster. One conflict of selection pressures frequently observed by experimental geneticists is that between natural and artificial selection. In this case we may if we wish regard the animals as competing for the favour of the experimenter, so that here competition leads to directional selection. A conflict of directional and centripetal selection may possibly occur in some natural populations through the agency of sexual selection.

In general, the varying selective forces will be exceedingly complex, so that for a discussion in quantitative terms, a simplified model of the process must be used. Robertson (1956) developed some of the consequences of two models in which intermediate phenotypes were favoured; the first involved centripetal selection on the basis of the phenotype itself, while the second involved genotypic selection of heterozygotes, the phenotype in this case being affected through its correlation with the genotype. Latter (1960) extended the theory of the first of these models. In populations at equilibrium both models lead to a similar relationship between fitness and phenotype, fitness being proportional to the square of the deviation from the mean. If the mean is shifted from the equilibrium value, the decline in average fitness under both models is proportional to the square of that change. For the model based on heterozygote selection, however, there is now a linear relation between fitness and deviation within the population.

Both models could be expected to lead to a limit being placed on response to directional selection, and in view of Robertson's ( 1960 ) theory of selection limits based on fixation it is of interest to consider 
the limits imposed by opposing natural selection. In this paper the effects of the model based on phenotypic selection on the limits to selection advance are considered. While this model can hardly be regarded as an adequate description of what happens in real populations, it may be of value as a first step towards a theory of the effect of opposing natural selection on the response to directional selection, and in the absence of a more general theory, may be useful as a rough guide to what will happen in practice.

\section{THE MODEL}

The character under consideration is taken to be a metric trait which, before centripetal selection operates, is normally distributed with mean $\mu$ and variance $\sigma_{p}^{2}$. To simplify the algebra, the scale is chosen so that the origin has maximum fitness under centripetal selection. The pattern of centripetal selection is that discussed by Haldane (1954) in which an individual with phenotypic value $x$ has a probability of survival proportional to $\exp \left\{-\frac{x^{2}}{2 \sigma_{f}^{2}}\right\}$, where $\sigma_{f}^{2}$ is a constant which is large for weak, and small for intense selection. It is then readily shown that among survivors the trait is normally distributed about a mean of $\frac{\sigma_{f}^{2}}{\sigma^{2}} \mu$ with variance $\frac{\sigma_{f}^{2}}{\sigma^{2}} \sigma_{p}^{2}$, where $\sigma^{2}=\sigma_{p}^{2}+\sigma_{f}^{2}$. If $I$ is the intensity of selection as measured by Haldane's (1954) criterion,

$$
I=\frac{1}{2} \log \left(I+\frac{\sigma_{p}^{2}}{\sigma_{f}^{2}}\right)+\frac{\mu^{2}}{2 \sigma^{2}},
$$

all logarithms in this paper being natural logarithms.

The average survival rate in such a population is seen to be a fraction $\exp \left\{-\frac{\mu^{2}}{2 \sigma^{2}}\right\}$ that of a population with mean at the optimum, which population may be denoted $\Pi_{0}$ for convenience. The intensity of selection in $\Pi_{0}$ is denoted $I_{0}$, as this will appear frequently.

The above results will obviously apply equally well to normally clistributed subgroups of this population.

It is not necessary for the present purpose that the trait itself be directly responsible for the centripetal selection. It may for instance be regarded as a measure of certain developmental processes which do affect fitness, but it must be phenotypically related to these processes, and not merely genetically.

Now it is assumed that following centripetal selection, which may be imagined as occurring through juvenile survival, directional selection occurs by truncation of the phenotypic distribution at a point which allows only a certain fixed proportion of survivors to breed. This scheme of selection has obvious relevance to artificial selection experiments, but is likely to be rather different from what would happen 
under a similar conflict of selective forces in natural populations. Nevertheless, the results should be similar to those obtained under more general conditions. The two sexes are assumed equivalent under all considerations above.

\section{GENETIC CONSEQUENCES}

The discussion is restricted to additive gene action at loci with two alleles, the genotypes being present in random mating proportions. Thus a single locus has genotypes $A_{1} A_{1}, A_{1} A_{2}$ and $A_{2} A_{2}$ present in proportions $p^{2}, 2 p q$ and $q^{2}$ respectively, where $p+q=\mathrm{I}$. The difference between homozygotes is $a$, which is small compared with the phenotypic standard deviation $\sigma_{p}$. The three genotypes are then normally distributed with variance very nearly $\sigma_{p}^{2}$, about means of $\mu+q a$, $\mu+\left(q-\frac{1}{2}\right) a$, and $\mu-p a$ respectively. Thus the proportions of the three genotypes among survivors of centripetal selection are

$$
\begin{aligned}
& A_{1} A_{1}: p^{2} \exp \left\{-\frac{\mathrm{I}}{2 \sigma^{2}}(\mu+q a)^{2}\right\} \\
& A_{1} A_{2}: 2 p q \exp \left\{\frac{\mathrm{I}}{2 \sigma^{2}}\left(\mu+\left(q-\frac{1}{2}\right) a\right)^{2}\right\} \\
& A_{2} A_{2}: q^{2} \exp \left\{-\frac{\mathrm{I}}{2 \sigma^{2}}(\mu-p a)^{2}\right\}
\end{aligned}
$$

These are readily manipulated into the form

$$
\begin{aligned}
& A_{1} A_{1}: p^{2} \\
& A_{1} A_{2}: 2 p q \exp \left\{\frac{\mu a+\left(q-\frac{1}{4}\right) a^{2}}{2 \sigma^{2}}\right\} \\
& A_{2} A_{2}: q^{2} \exp \left\{\frac{2 \mu a+(2 q-1) a^{2}}{2 \sigma^{2}}\right\}
\end{aligned}
$$

The means of these subgroups are respectively $\frac{\sigma_{f}^{2}}{\sigma^{2}}(\mu+q a), \frac{\sigma_{f}^{2}}{\sigma^{2}}$ $\left\langle\mu+\left(q-\frac{1}{2}\right) a\right)$ and $\frac{\sigma_{f}^{2}}{\sigma^{2}}(\mu-p a)$, and the variance is $\frac{\sigma_{f}^{2}}{\sigma^{2}} \sigma_{p}^{2}$. Thus as shown by Haldane (1930), the relative probabilities of inclusion among parents following directional selection are

$$
\mathrm{I}+\frac{\tau \sigma_{f}}{\sigma_{p} \sigma} q a ; \quad \mathrm{I}+\frac{\tau \sigma_{f}}{\sigma_{p} \sigma}\left(q-\frac{1}{2}\right) a ; \quad \mathrm{I}-\frac{\tau \sigma_{f}}{\sigma_{p} \sigma} p a
$$

or the respective genotypes, where $\tau$ is the standardised selection 
differential. Putting $k=\frac{\tau \sigma_{f}}{\sigma_{p} \sigma}$, the relative frequencies of the three genotypes among parents are

$$
\begin{aligned}
& A_{1} A_{1}: p^{2}(\mathrm{I}+k q a) \\
& A_{1} A_{2}: 2 p q\left(\mathrm{I}+k\left(q-\frac{1}{2}\right) a\right) \exp \left\{\frac{\mu a+\left(q-\frac{1}{4}\right) a^{2}}{2 \sigma^{2}}\right\} \\
& A_{2} A_{2}: q^{2}(\mathrm{I}-k p a) \exp \left\{\frac{2 \mu a+(2 q-\mathrm{I}) a^{2}}{2 \sigma^{2}}\right\}
\end{aligned}
$$

When $\mu=k=o$ (i.e. centripetal selection towards the population mean),

$$
\Delta p \approx \frac{p q a^{2}}{8 \sigma^{2}}(p-q)
$$

as shown by Robertson (1956).

When $\mu=0, k>0$, it is easily seen that

$$
\Delta p \approx \frac{p q a}{2 \sigma}\left[\frac{\tau \sigma_{f}}{\sigma_{p}}+\frac{(p-q) a}{4^{\sigma}}\right]
$$

Thus unless centripetal selection is very strong $\left(\frac{\sigma_{f}}{\sigma_{p}}\right.$ very small $)$ or directional selection very weak ( $\tau$ very small) the direction of change in gene frequency is determined by directional selection, and it is necessary to consider the situation when $\mu$ is some distance from the optimum. When $\mu$ is much larger than $a$, since $a$ is much smaller than. $\sigma_{p}$, the genotypic ratios among parents are approximately:

$$
\begin{aligned}
& A_{1} A_{1}: p^{2}(\mathrm{I}+k q a) \\
& A_{1} A_{2}: 2 p q\left(\mathrm{I}+k\left(q-\frac{1}{2}\right) a\right)\left(\mathrm{I}+\frac{\mu a}{2 \sigma^{2}}\right) \\
& A_{2} A_{2}: q^{2}(\mathrm{I}-k p a)\left(\mathrm{I}+\frac{\mu a}{\sigma^{2}}\right)
\end{aligned}
$$

whence

$$
\Delta p \approx \frac{1}{2} p q a\left[k-\frac{\mu}{\sigma^{2}}\right]
$$

If there is to be an equilibrium, $\Delta p=0$, and the condition for this is

$$
\mu=k \sigma^{2}
$$

Thus, if $\mu^{*}$ is the mean at equilibrium

$$
\mu^{*}=\frac{\tau \sigma_{f} \sigma}{\sigma_{p}}
$$


This conflict of selective pressures may thus lead to an equilibrium at which intermediate gene frequencies may be maintained. The model, however, gives no information as to what these gene frequencies may be, as they will depend on the frequencies and effects of alleles at all loci affecting the trait.

If this equilibrium population is denoted $\Pi^{*}$, the survival rate in $\Pi^{*}$ is a fraction $\exp \left\{-\frac{\tau^{2} \sigma_{f}^{2}}{2 \sigma_{p}^{2}}\right\}$ that in $\Pi_{0}$. This is conveniently expressed by comparing $I^{*}$, the intensity of selection in $\Pi^{*}$. If $I_{0}$ is not too large,

and

$$
I_{0} \approx \frac{\sigma_{p}^{2}}{2 \sigma_{f}^{2}}
$$

$$
I^{*}-I_{\rho} \approx \frac{\tau^{2}}{4 I_{0}}
$$

For suitable values of $\tau$ and $I_{0}$ this increase in the intensity of selection may be quite large. For example, if $I_{0}$ is about 0.05 and half the survivors breed, so that $\tau$ is about 0.8 ,

$$
I^{*}-I_{0} \approx 3
$$

In this case $\Pi^{*}$ may be in danger of dying out. It is worth noting that only when $I_{0}$ is low can directional selection move the mean very far from the optimum, so that greater decreases in survival rate occur when the selected trait is less closely associated with fitness.

\section{ARTIFICIAL SELECTION}

If under natural conditions centripetal selection of the type considered here operates, the model is clearly relevant to the results of selection experiments. It will be noted that the total advance which can be achieved is $\frac{\tau \sigma_{f} \sigma}{\sigma_{p}^{2}}$ times the phenotypic standard deviation in the original population. This result is quite independent of genetic parameters, and draws attention to the fact that it is assumed no other agency, such as fixation of all additive genetic variability, prevents the population from reaching this equilibrium. If $I_{0}$ is not too large, this maximum gain is about $\frac{\tau}{2 I_{0}}$ times the initial standard deviation. Thus if $I_{0}$ is between 0.05 and 0.1 , this maximum gain is about $5 \tau$ to Io $\tau$ times $\sigma_{p}$.

Another point of interest here arises from a result of Latter (1960). He showed that if centripetal selection of this type were in equilibrium with recurrent mutation, then

$$
h^{2}=\frac{2 n v}{I_{0}}
$$


where $n$ is the number of loci controlling the trait and $v$ is the mean mutation rate at these loci. Thus if we denote the initial response by $\Delta G$, so that

$$
\Delta G=\tau h^{2} \sigma_{p}
$$

then $\frac{\mu^{*}}{\Delta G}=\frac{\sigma_{f} \sigma}{h^{2} \sigma_{p}^{2}}$, and when $I_{0}$ is small this is roughly $\frac{I}{4^{n v}}$. Thus if $v$ is of the order of $10^{-5}$, and $\frac{\mu^{*}}{\Delta G}$ is about 20, we must assume about 1250 loci affecting the trait. This conclusion may, however, be vitiated by the fact that the equation for $h^{2}$ above applies only to loci with effects large enough for centripetal selection to determine the equilibrium with recurrent mutation. If the gene effects are very small, the equilibrium gene frequency will be determined by the forward and backward mutation rates, and no conclusions can safely be drawn.

The shape of the response curve under this system of selection can also be easily found. Since

$$
\begin{aligned}
\Delta p & =\frac{1}{2} p q a\left(k-\frac{\mu}{\sigma^{2}}\right) \\
\Delta \mu & =\Sigma a \Delta p \\
& =\left(k-\frac{\mu}{\sigma^{2}}\right) \sigma_{g}^{2}
\end{aligned}
$$

Assuming that $\sigma_{g}^{2}$ is not greatly altered during the course of selection, this difference equation can be solved to give

$$
\mu_{t}=\frac{\tau \sigma_{f} \sigma}{\sigma_{p}}\left[\mathrm{I}-\left(\mathrm{I}-\frac{\sigma_{g}^{2}}{\sigma^{2}}\right)^{t}\right]
$$

where $\mu_{t}$ is the mean after $t$ generations of selection. For small values of $I_{0}$ this is approximately

$$
\mu_{t}=\frac{\tau \sigma_{p}}{2 I_{0}}\left[\mathrm{I}-\left(\mathrm{I}-2 I_{0} h^{2}\right)^{t}\right]
$$

It is noteworthy that the response curve for Robertson's (1960) model with additive gene action is given by

$$
\mu_{t}=2 \mathcal{N} \Delta G\left[\mathrm{I}-\left(\mathrm{I}-\frac{\mathrm{I}}{2 \mathcal{N}}\right)^{t}\right]
$$

where $\mathcal{N}$ is the effective population size. The form of the response curve is the same in both cases, though the parameters involved are very different. The "half-life" $H$, for Robertson's model, that is, the time taken to reach half the total advance was given by

$$
H \approx \mathrm{I} \cdot 4 \mathcal{N} \text {. }
$$


For the present model, it is easily seen that

or roughly,

$$
H=\frac{\log \left(\frac{1}{2}\right)}{\log \left(\mathrm{I}-\frac{\sigma_{g}^{2}}{\sigma^{2}}\right)}
$$

$$
H \approx \frac{0 \cdot 35}{I_{0} h^{2}}
$$

The assumption that $\sigma_{g}^{2}$ does not alter during selection is made for convenience, though experimental results suggest it is not badly wrong. However, in view of the fact that selection on crosses between plateaued lines may yield further gains, it is useful to consider the effect of decline of genetic variance. No exact analysis has been made, but a rough approach is possible through the assumption that genetic variance is reduced by a fraction $\frac{\mathrm{I}}{2 \mathcal{N}}$ every generation. The result is that the difference equation becomes

$$
\Delta \mu_{t}=\left(k-\frac{\mu_{t}}{\sigma^{2}}\right) \sigma_{B}^{2}\left(\mathrm{I}-\frac{\mathrm{I}}{2 \mathcal{N}}\right)^{t}
$$

No solution of this equation has been found. But if it is rewritten as a differential equation, it becomes

$$
\frac{d \mu}{d t}=\left(k-\frac{\mu}{\sigma^{2}}\right) \sigma_{s}^{2}\left(1-\frac{1}{2 \mathcal{N}}\right)^{t}
$$

and this has the solution

$$
-\frac{\sigma^{2}}{\sigma_{g}^{2}} \log \left[k \sigma_{g}^{2}-\frac{\mu \sigma_{g}^{2}}{\sigma^{2}}\right]=\frac{\left(\mathrm{I}-\frac{\mathrm{I}}{2 \mathcal{N}}\right)^{t}}{\log \left(\mathrm{I}-\frac{\mathrm{I}}{2 \mathcal{N}}\right)}+\theta
$$

where $\theta$ is the constant of integration. Using the condition $\mu=0$ when $t=0$ to evaluate $\theta$,

$$
\mu=\frac{\tau \sigma_{f} \sigma}{\sigma_{p}}\left[\mathrm{I}-\exp \left\{\frac{\frac{\sigma_{g}^{2}}{\sigma^{2}}}{\log \left(\mathrm{I}-\frac{\mathrm{I}}{2 \mathcal{N}}\right)}\left(\mathrm{I}-\left(\mathrm{I}-\frac{\mathrm{I}}{2 \mathcal{N}}\right)^{t}\right)\right\}\right]
$$

When $I_{0}$ is small and $\mathcal{N}$ large, this is roughly

$$
\mu=\frac{\tau \sigma_{p}}{2 I_{0}}\left[\mathrm{I}-\exp \left\{-4 N I_{0} h^{2}\left(\mathrm{I}-\left(\mathrm{I}-\frac{\mathrm{I}}{2 \mathcal{N}}\right)^{t}\right)\right\}\right]
$$


The asymptotic limit of response is now given by

$$
\mu^{*}=\frac{\tau \sigma_{f} \sigma}{\sigma_{p}}\left[\mathrm{I}-\exp \left\{\frac{\frac{\sigma_{g}^{2}}{\sigma^{2}}}{\log \left(\mathrm{I}-\frac{\mathrm{I}}{2 \mathcal{N}}\right)}\right\}\right]
$$

As $I_{0} \rightarrow \theta, \mu^{*} \rightarrow 2 \mathcal{N} \Delta G$, as given by Robertson (1960). There seems to be no simple general expression for the half-life, though it can of course be worked out in any particular case. When $\mathcal{N}$ is large and $I_{0}$ very small so that $\mathcal{N} I_{0}$ is small, we have as a very rough approximation

$$
\mu^{*} \approx 2 \mathcal{N} \Delta G\left(\mathrm{I}-2 \mathcal{N} I_{0} h^{2}\right) .
$$

This indicates that for small values of $\mathcal{N}$, provided $I_{0}$ is small, Robertson's model will not greatly overestimate the selection limit. However, for moderate values of $\mathcal{N}$, the limit will fall well below $2 \mathcal{N} \Delta G$, especially for characters with high heritabilities.

The relation between the selection limit and the various parameters are shown in figs. I and 2. Fig. I gives the limit obtained in units of

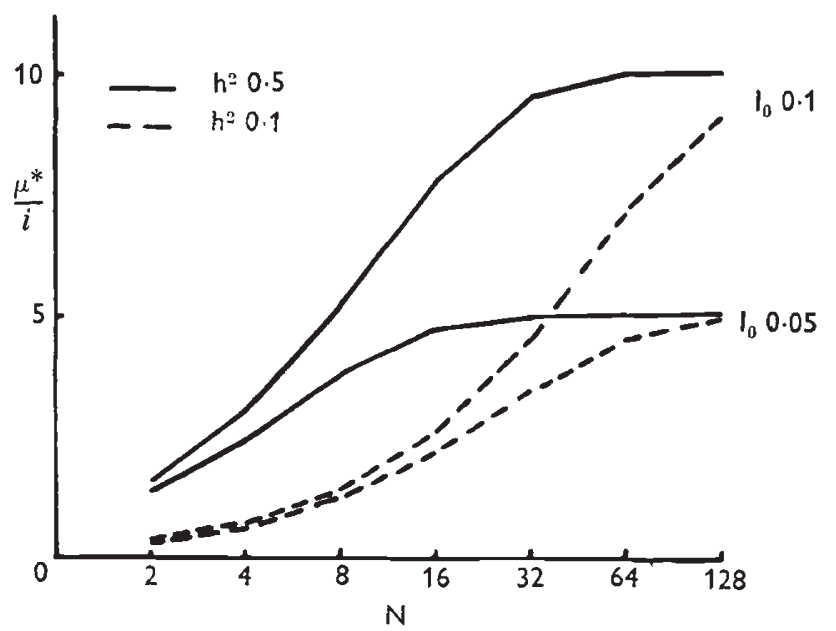

Fis. 1.-Relations between selection limits $\left(\mu^{*}\right)$, selection differential $(i)$, intensity of natural selection $\left(I_{0}\right)$, effective population size $(\mathcal{N})$ and heritability $\left(h^{2}\right)$.

$\tau \sigma_{p}$, the selection differential. It can be seen that for high heritability characters, a very high proportion of the maximum attainable response is obtained when $\mathcal{N}$ is about 25. The effect of effective size is greater for low heritabilities, if $\tau$ and $I_{0}$ are similar, for the obvious reason that when $h^{2}$ is low it takes longer to reach the limit, so that chance fixation has a longer time in which to operate.

Fig. 2 shows the selection limit in terms of the initial gain, $\Delta G$. Since the initial response is lower for low $h^{2}$, limits of the same value in terms of $\sigma_{p}$ are higher for low $h^{2}$, so that the curves for $h^{2}=0 \cdot I$ 
are above those for $h^{2}=0.5$, but this does not mean that absolute gains are greater. Fig. 2 shows many of the features of fig. I. The line for $I_{0}=0$ gives the response as $2 \mathcal{N} \Delta G$, as follows from the model of Robertson (1960). It can be seen that this will overestimate the limit more for high than low heritability, and more for intense than for weak natural selection. Furthermore, these results become more pronounced as $\mathcal{N}$ increases.

The points marked on fig. 2 refer to the results of selection experiments carried out on Drosophila and on poultry. The four points AF, $\mathrm{RF}, \mathrm{AO}$, and RO refer to the limits found by McBride (1959) in

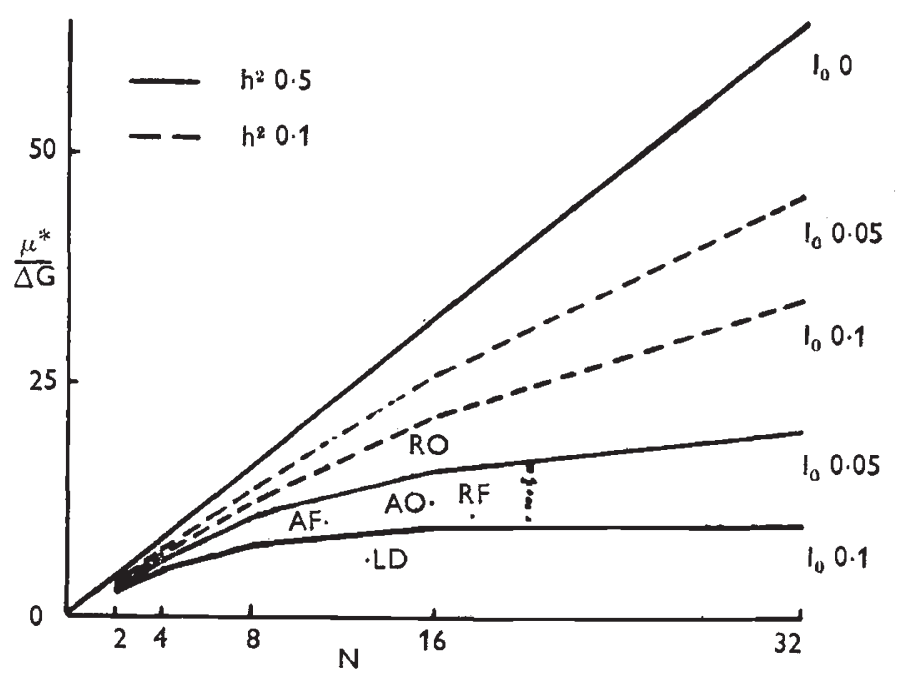

Frg. 2.- Selection limit $\left(\mu^{*}\right)$ in terms of initial response $(\Delta G)$ for different heritabilities $\left(h^{2}\right)$, effective population sizes $(\mathcal{N})$ and intensities of natural selection $\left(I_{0}\right)$. The points refer to experimental data. For details see text.

four of his selected lines. Two of his lines are omitted because of the occurrence of a single recessive gene, scabrous, with a large effect on bristle number, for which McBride's flies were selected. The effective sizes have been calculated from inbreeding coefficients, and $\Delta G$ is the average gain over the first five generations. Except for RO, the lines would suggest a value of $I_{0}$ of about $0 \cdot 075$. The exceptional line RO went through an inbreeding bottleneck early, and its initial response was considerably lower than in the other three lines.

The points at $\mathcal{N}=20$ refer to the limits attained by the ten lines of Clayton and Robertson (1957). The limits are perhaps less reliable here, as some lines were continued for 30 and others for only 20 generations. No inbreeding coefficients were available, so $\mathcal{N}$ was taken as half the total number of parents $(20 \hat{\jmath} \hat{o}+20 q$ q $)$. McBride's lines which were pedigree mated had $\mathcal{N}$ equal to about $\frac{3}{4}$ of the number of parents in the individual selection lines (the $F$ lines were selected on family and individual index), and to allow roughly for the effect of mass 
mating in Glayton and Robertson's lines, $\mathcal{N}$ was taken to be reduced to half the number of parents. These lines are also in rough agreement with a value of $I_{0}$ between 0.05 and 0.1 .

Of particular interest for the present purpose is the remaining point on fig. 2, labelled LD. This represents the result of the shank length experiment in poultry carried out by Lerner and Dempster (1951). From their table I, $\Delta G$ is taken as $0 \cdot 19$, and from table I of Lerner (1954) $\mu^{*}$ is estimated at about $1 \cdot 33$, so that $\frac{\mu^{*}}{\Delta G}$ is about 7 . From Lerner and Dempster's table 2, $\mathcal{N}$ is estimated at about I 2 and $\sigma^{2}{ }_{p}$ about $0 \cdot 24$. From the text of their paper $h^{2}$ is taken as about $0 \cdot 4$. Using these figures $I_{0}$ is estimated as about $0 \cdot 17$. The particular value of this experiment is that the authors have given an index of fitness for various generations which is more or less equivalent to average survival rate. From the relation between survival rate $\left(\lambda_{t}\right)$ and mean $\left(\mu_{t}\right)$ given in an earlier section,

$$
-\log \lambda_{t}=\text { const }+\frac{1}{2 \sigma^{2}} \mu_{t}^{2}
$$

so that $I_{0}$ can be estimated by regression of $-\log \lambda_{t}$ on $\mu_{t}^{2}$. It turns out that $I_{0}$ as estimated in this way is roughly $0 \cdot 125$, so that $I_{0}$ as estimated from $\frac{\mu^{*}}{\Delta G}$ seems to be an overestimate. It may be expected that this will also be true for the other cases discussed.

\section{DISCUSSION}

The present treatment is essentially an extension of the work of Robertson (1956) and Latter (1960). Detailed analysis is given only to additive gene action in large populations, though some attention has been given to chance fixation. It is therefore unlikely that the model would give accurate descriptions of experimental selection responses, even should the model be adequate in other respects. However, the results will bear discussion in a general way. First, if selection pressures do act as assumed here, an equilibrium may be reached at which the survival rate is low. Under not inconceivable conditions, $I^{*}-I_{0}$ may be as great as 3 , or in other words, the survival rate in $\Pi^{*}$ may be only one-twentieth that in $I I_{0}$.

As this study was undertaken following some remarks of $\mathrm{Dr}$ J. M. Rendel on the Irish elk, this matter may appropriately be discussed here. It is often asserted that the species became extinct because sexual selection for weight and antler size in males led to such large antlers that the animals were unable to escape from their predators. The present analysis suggests that if indeed these were the sole forces acting, fitness may decline to such a low value that extinction is virtually certain. The difficulty consists still, as it always has, in accepting the argument that the action of such selective forces can 
proceed unimpeded by other factors. Mather (I $96 \mathrm{I}$ ) has recently expressed doubt in this argument, and many will share his view. If such conflicts of selective pressures occurred at all frequently in nature, it might be expected that cases would be found where the optimum phenotype was some distance from the mean, unless indeed extinction is the fate of those populations unable to resolve the conflict. Relatively few studies of this type have been made, but these have shown no marked difference between mean and optimum (see Haldane, 1954).

In an experimental population of the domestic fowl, Lerner and Gunns (1952) found that maximum fitness was at a phenotype for egg weight some distance below the mean. This flock had been subjected to intermittent selection for high egg weight. It is interesting that fig. I of these authors does not show the linear relation between fitness and phenotype predicted by Robertson (1956) from the " homeostatic" model, but a pattern more like that assumed in this paper.

In artificial selection programmes, the conflict of selective forces wiil set the limit to response only if no other forces prevent this limit from being attained. Though a few results, such as that of Bell, Moore and Warren (1957) have attributed plateau formation to loss of additive genetic variance, this does not seem very common, so that opposing selection is at least in part responsible for cessation of response. The problem is complicated by the fact that selection on crosses between plateaued lines may lead to further gains.

Though it is not suggested that the mechanism discussed here is the sole (or even main) cause of plateau formation, the comparisons of experimental limits with the theory developed here do not lead to wildly unlikely results. It would seem that the limits are somewhat lower than would be predicted if $I_{0}$ were known, so that the value of $I_{0}$ of about 0.075 for bristle number in $D$. melanogaster may be somewhat too high. It is to be expected that selection of heterozygotes or balanced blocks of polygenes would reduce the limit below that predicted from the model.

That centripetal selection for bristle number is not negligible has been well shown by Mather (Ig6r), though his results do not furnish an estimate of the intensity of selection. In connection with these Drosophila melanogaster experiments we should note that at the limit

$$
I^{*}-I_{0} \approx I_{0}\left(\frac{\mu^{*}}{\sigma_{p}}\right)^{2}
$$

and since $\mu^{*}$ was of the order of 5 to Io times $\sigma_{p}$, taking $I_{0}$ as 0.075 would give $I^{*}-I_{0}$ about 2 to 8 . This would mean that at the limit the survival rate would lie between about one tenth and one threethousandth that in $\Pi_{0}$. This shows how rapidly, after a certain stage has been reached, fitness falls off with increased gain for the present model. Unfortunately no quantitative data on survival rates have been obtained in these experiments. 
In terms of the model there would be a close negative correlation between fitness and the amount of gain. In practice we should expect this relation to be obscured. For on the present assumptions, increase in $\mathcal{N}$ increases the limit, and hence decreases fitness, whereas a greater inbreeding depression of fitness should occur at low values of $\mathcal{N}$.

It has perhaps been sufficiently emphasised that the present model does not take account of natural selection by other agencies than the deviation of phenotypes from the optimum. However, even allowing that this mechanism does act, we may question the validity of the functional relation between fitness and deviation. The function has obviously been chosen so as to leave the distribution of survivors' phenotypes normal, i.e. for mathematical convenience. Even if this should be a good approximation when $u$ is near the optimum it would not necessarily be so when $u$ is a long way from the optimum, especially when it is remembered that the genetic constitution of the population will then be very different. Problems of scaling will enter too, but there is little point in elaborating further the defects of the model, as these should be apparent. It may suffice to express the opinion that part of the natural selection usually found to oppose directional selection is based on higher fitness of intermediate phenotypes, perhaps partly through developmental canalisation (Waddington, 1957).

The actual situation is extremely complicated, and no simple model is likely to be adequate. It may then be necessary to justify the detailed analysis of such a model.

First, though this is of only limited value, it is of interest to know the consequences of certain sets of assumptions. Especially in evolutionary discussions many of the arguments advanced are not based on any quantitative foundation, and simple models allowing quantitative statements are better than none at all. (This is not intended to denigrate the extensive theory applying to evolutionary changes in frequencies of single genes, but to situations such as that of the Irish elk.)

Secondly, though there have been considerable recent advances in the static genetic analysis of metric traits (see Kempthorne, I957) the theory of their dynamics is still in its infancy, and simple models are probably all that can yet be successfully managed. There is a dearth of quantitative predictions against which experimental geneticists can test their results, and additions are useful, even if the models are oversimplified. The existence of such a set of predictions will usually lead to critical experimentation, and this will give indications of where the theory is at fault. This in turn will give a basis for further elaboration of models. Thus provided our thinking is not rigidly conditioned by the existence of such a model, we are likely to achieve a series of successively better approximations to reality.

It is then in order to provide, as it were, a starting value for these approximations that the present analysis has been carried out. 


\section{SUMMARY}

A model for the conflict of directional selection with centripetal selection based on inferior fitness of extreme phenotypes is analysed. It is shown that a plateau may be formed at which genetic variance is present and fitness is reduced. Approximate expressions are given for the selection limit and the fitness at that limit. The form of the response curve is shown to be the same as that when limits are caused by fixation of variability.

\section{REFERENCES}

BELL, A. E., MOORE, C. H., AND WARREN, D. C. 1957. Genetic changes in closed populations of $D$. melanogaster under continuous selection. Genetics, 42, 36o.

CLAYTON, G. A., AND ROBERTSON, A. I957. An experimental check on quantitative genetical theory. II. The long term effects of selection. 7. Genet., 55, i 52-i 70.

HALDANE, J. B. S. I930. A mathematical theory of natural and artificial selection. VII. Selection intensity as a function of mortality rate. Proc. Camb. Phil. Soc., $27,131-136$.

haldane, J. B. s. 1954. The measurement of natural selection. Proc. gth. Int. Congr. Genet., part I, $480 \cdot 487$.

KARN, M. A., AND PENROSE, L. S. I95I. Birth weight and gestation time in relation to maternal age, parity, and infant survival. Ann. Eugen., I6, I47-164.

Kempthorne, oscar. 1957. An Introduction to Genetic Statistics. John Wiley and Sons, New York.

LACK, D. 1954. The evolution of reproductive rates, in Evolution as a Process. Allen and Unwin, London.

LATTER, B. D. H. 1960. Natural selection for an intermediate optimum. Aust. $\mathcal{F}$. Biol. Sci., 13, 30-35.

Lerner, I. M. I 954. Genetic Homeostasis. Oliver and Boyd, Edinburgh.

LERNER, I. M., AND DEMPSTER, E. R. I95I. Attenuation of genetic progress under continued selection in poultry. Heredity, 5, 75-94.

LERner, I. M., AND GUNNo, C. A. 1952. Egg size and reproductive fitness. Poult. Sci., 31, 537-544.

MATHer, K. I 96 I. Competition and cooperation. Symposia of the Society for Experimental Biology. XV. Mechanisms in biological competition. r $96 \mathbf{r}$.

McBRIDE, G. 1959. Ph.D. Thesis. University of Edinburgh.

RENDEL, J. M. I943. Variations in the weights of hatched and unhatched ducks' eggs. Biometrika, 33, 48-58.

ROBERTSON, A. 1956. The effect of selection against extreme deviants based on deviation or on homozygosis. 7. Genet., 54, 236-248.

robertson, A. 1960. A theory of limits in artificial selection. Proc. Roy. Soc. B., I53, 234-249.

waddington, c. H. 1957. The Strategy of the Genes. Allen and Unwin, London. 